\title{
Photometric structure of polar-ring galaxies
}

\author{
V. P. Reshetnikov ${ }^{\star}$
}

\author{
Astronomical Institute of St. Petersburg State University, 198504 St. Petersburg, Russia \\ Isaac Newton Institute of Chile, St. Petersburg Branch, Russia
}

Received 3 June 2003 / Accepted 21 August 2003

\begin{abstract}
The results of $B, V, R$ surface photometry of three polar-ring galaxies (PRGs) - A 0017+2212, UGC 1198, UGC 4385 - are presented. The data were acquired at the 6-m telescope of the Special Astrophysical Observatory of the Russian Academy of Sciences. It was shown that all three galaxies are peculiar late-type spirals in the state of ongoing interaction or merging.

We discuss available photometric properties of the PRGs with spiral hosts and consider the Tully-Fisher relation for different types of PRGs. In agreement with Iodice et al. (2003), we have shown that true PRGs demonstrate $\sim 1 / 3$ larger maximum rotation velocities than spiral galaxies of the same luminosity. Peculiar objects with forming polar structures satisfy, on average, the Tully-Fisher relation for disk galaxies but with large scatter.
\end{abstract}

Key words. galaxies: individual: A 0017+2212, UGC 1198, UGC 4385 - galaxies: photometry - galaxies: formation galaxies: structure

\section{Introduction}

Polar-ring galaxies (PRGs) are peculiar objects composed of a central component surrounded by an outer nearly perpendicular ring or disk, made up of gas, stars and dust (Whitmore et al. 1990, Polar Ring Catalog = PRC). It is generally agreed that the formation of the polar rings is the result of some kind of "secondary event" - external accretion or a merger. Recent numerical simulations have confirmed that both scenarios are robust, and result in realistic polar structures (Bekki 1997, 1998; Reshetnikov \& Sotnikova 1997; Bournaud \& Combes 2003).

PRGs are ideal laboratories to study the shape of their dark matter haloes, and gain insight into the galaxy formation through late infall, because of the high inclination of their outer structures and the evidence of very recent or occuring gas infall. PRC lists more than 100 PRGs and candidates for PRGs. Unfortunately, only a small fraction of them are investigated. The last years have seen renewed interest in PRG studies, triggered by the wonderful data acquired during the HST heritage program for the polar-ring galaxy NGC 4650A (Kinney et al. 1999).

In this article we present the results of photometric observations of three possible candidates for PRGs from the PRC. One of the galaxies - UGC 4385 - is a true, kinematicallyconfirmed polar-ring galaxy with a spiral host (Reshetnikov \& Combes 1994). Two other galaxies (A 0017+2212 and UGC 1198) are almost uninvestigated peculiar objects. In the last section of the paper we discuss the available data on the

\footnotetext{
* e-mail: resh@astro.spbu.ru
}

photometric structure of PRGs and re-examine the Tully-Fisher relation for various groups of PRGs.

\section{Observations and reductions}

The photometric observations were carried out in 1992 and 1996 in the prime focus of the 6-m telescope of the Special Astrophysical Observatory of the Russian Academy of Sciences, using a CCD detector with $520 \times 580$ pixels, each $18 \times 24 \mu \mathrm{m}\left(0{ }^{\prime} 15 \times 0{ }^{\prime}\right.$ '20) (Borisenko et al. 1991). The data were acquired with standard Johnson $B, V$ and Cousins $R$ filters. Photometric calibration was accomplished using repeated observations of standard stars from the Landolt (1983) and Smith et al. (1991) lists. The seeing during the observations was 1". 8 (A 0017+2212, UGC 4385) and 1'.'0 for UGC 1198. A $\log$ of observations is given in Table 1 (extinction corrected sky brightness (in mag $\operatorname{arcsec}^{-2}$ ) in each frame is presented in the last column of the table). Reduction of the CCD data has been performed in the standard manner using the ESOMIDAS $^{1}$ package.

The total magnitudes of galaxies found by us from the multiaperture photometry (see Table 2) are in good agreement with the $\mathrm{NED}^{2}$ data $\left(B_{\mathrm{T}}=16.75,14.80 \pm 0.16,14.51 \pm 0.18\right.$ for A $0017+2212$, UGC 1198, UGC 4385 correspondingly). The mean difference between our and NED total magnitudes is $0.07 \pm 0.15(\sigma)$.

\footnotetext{
${ }^{1}$ MIDAS is developed and maintained by the European Southern Observatory.

${ }^{2}$ NASA/IPAC Extragalactic Database.
} 
Table 1. Observations.

\begin{tabular}{cccccc}
\hline \hline Object & Data & $\begin{array}{c}\text { Band- } \\
\text { pass }\end{array}$ & $\begin{array}{c}\text { Exp } \\
(\mathrm{s})\end{array}$ & $\begin{array}{c}Z \\
\left({ }^{\circ}\right)\end{array}$ & $\begin{array}{c}\text { Sky } \\
\text { mag. }\end{array}$ \\
\hline A 0017+2212 & Nov. 1992 & $R$ & 300 & 31 & 19.6 \\
(PRC C-2) & & $R$ & 300 & 30 & 19.6 \\
& & $V$ & 720 & 29 & 20.0 \\
& & $B$ & 900 & 27 & 20.5 \\
& & $B$ & 900 & 25 & 20.5 \\
UGC 1198 & \multirow{2}{*}{ Nov. 1992 } & $R$ & 300 & 42 & 20.4 \\
(PRC C-12) & & $V$ & 600 & 42 & 20.9 \\
& & $B$ & 900 & 42 & 21.8 \\
& & $B$ & 900 & 42 & 21.8 \\
UGC 4385 & May 1996 & $R$ & 300 & 33 & 20.6 \\
(PRC C-27) & & $V$ & 600 & 32 & 21.2 \\
& & $B$ & 900 & 31 & 22.1 \\
\hline
\end{tabular}

\section{Results and discussion}

\section{1. $A 0017+2212(P R C$ C-2)}

\subsubsection{General structure}

The $B$-band image is displayed in Fig. 1; Fig. 2 shows $V$-band contour map. The galaxy demonstrates a multicomponent structure. The central part of A $0017+2212$ is elongated along the position angle close to $0^{\circ}$ (north-south direction). The central body is surrounded by a narrow inclined (by about $30^{\circ}$ ) ring-like structure with angular diameter $\approx 20^{\prime \prime}$ and apparent axial ratio $b / a=0.4$. Two bright knots are located at the edges of the ring (Figs. 1 and 4). The central body and the ring are embedded in an asymmetric extended envelope. A small edgeon galaxy is located $45^{\prime \prime} \mathrm{SW}$ of the PRC object.

Figures $3 a, c$ show the surface brightness profiles along the apparent major axis of the ring $\left(\mathrm{PA}=43^{\circ}\right)$ and along the major axis of the central part of the galaxy $\left(\mathrm{PA}=13^{\circ}\right)$. In the major axis profile the ring is seen as two symmetrical maxima at $r \approx \pm 10^{\prime \prime}$. Excluding the regions of the ring projection, the general surface brightness distribution may be approximated by that of an exponential disk. In the $R$ passband the disk characteristics are: the central surface brightness $\mu_{R, 0}(0)=19.6^{3}$, exponential scalelength $h=3^{\prime \prime}$ at $\mathrm{PA}=13^{\circ}$ (or $h=4^{\prime \prime}$ at $\mathrm{PA}=43^{\circ}$ ). In the $B$-band the extrapolated central surface brightness of the disk is $\mu_{B, 0}(0)=20.6$. This value is about $1^{\mathrm{m}}$ brighter than that of the disks of normal bright spirals (Freeman 1970). Such bright galactic disks are usual for interacting systems (Reshetnikov et al. 1993).

Figures 3b,d display the observed color indices along two cuts. Both profiles show strong color gradients: the central regions of the galaxy are relatively red $\left((B-V)_{0} \approx+0.7\right)$, while the outer ones are blue $\left((B-V)_{0} \approx+0.4\right)$.

\footnotetext{
${ }^{3}$ Subscript "0" means value corrected for Galactic absorption according to Schlegel et al. (1998).
}

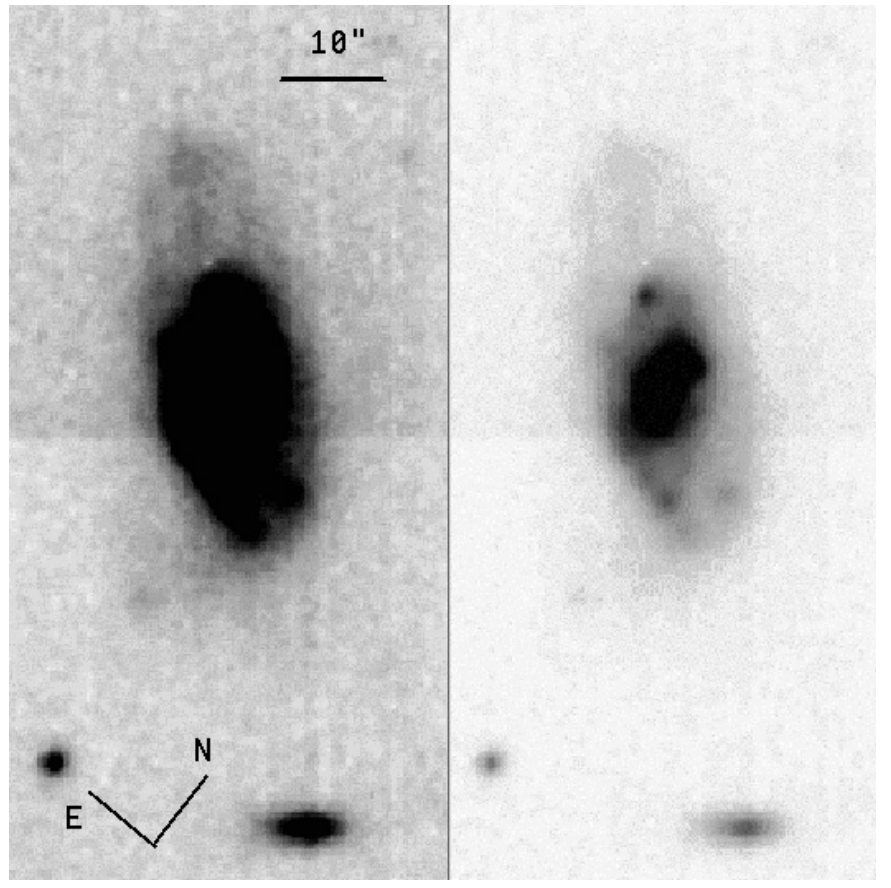

Fig. 1. $B$-band image of A $0017+2212$ displayed at two different contrasts. Each image size is $42^{\prime \prime} \times 85^{\prime \prime}$.

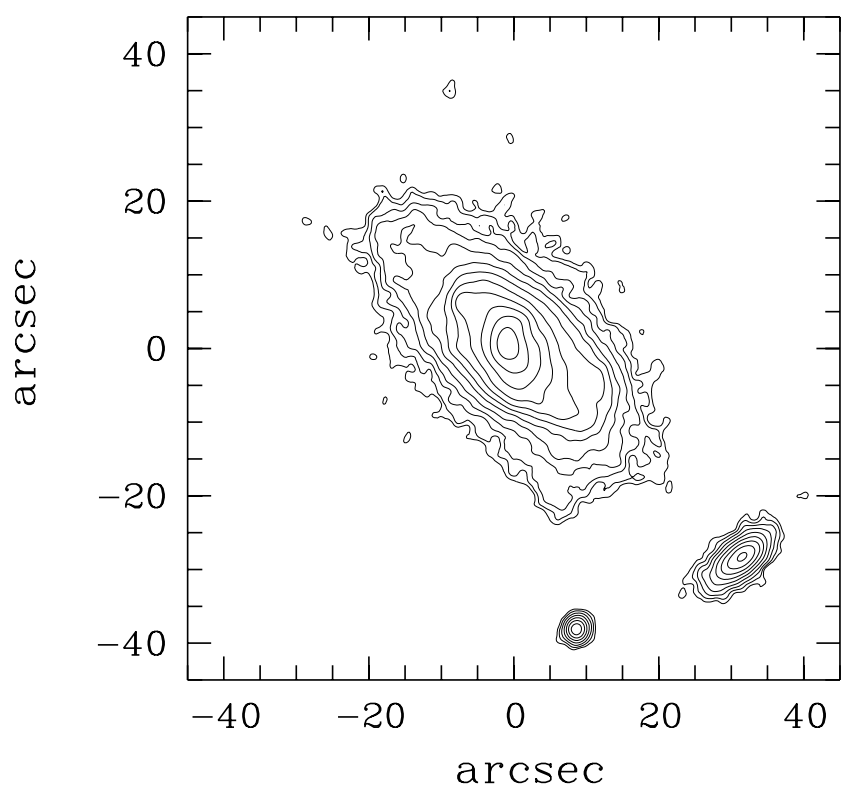

Fig. 2. Isophotal contour image of A $0017+2212$ in the $V$ passband. The faintest contour is $25.7 \mathrm{mag} \operatorname{arcsec}^{-2}$, isophotes step $-0 .{ }^{\mathrm{m}} 44$. North is at the top, east to the left.

From optical colors (see Buta et al. 1994; Buta \& Williams 1995) and the exponential surface brightness distribution we can preliminary classify the galaxy as an Sc/Scd spiral.

\subsubsection{Ring}

The suspected ring looks very narrow - with the ratio of radial extent to mean radius about $20 \%$ (Figs. 1 and 4 ). The total apparent magnitude of the ring is $B=19^{\mathrm{m}}$, or the ring contribution to the total luminosity of A $0017+2212$ is $\approx 1 / 9$. The mean 

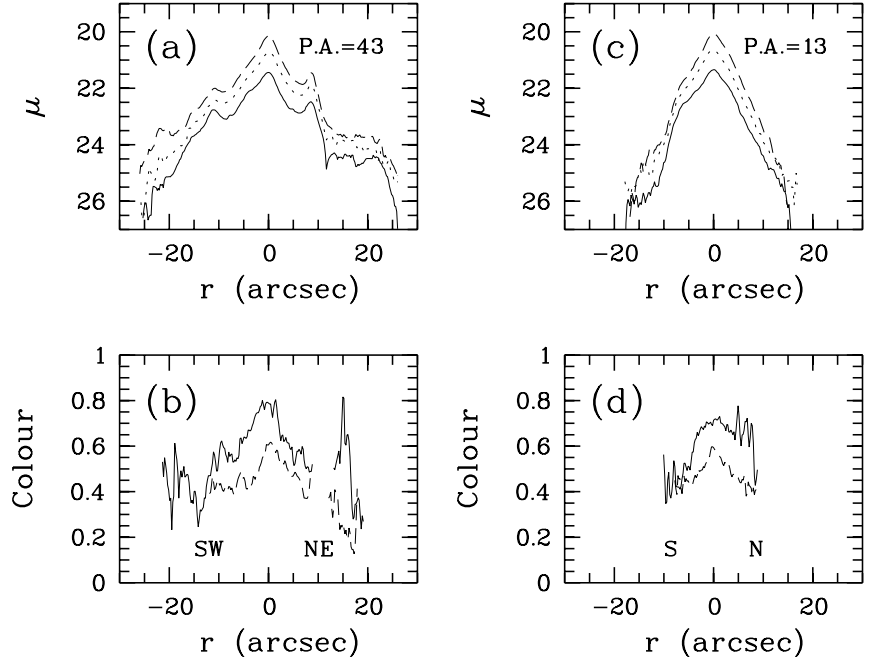

Fig. 3. Photometric profiles for A $0017+2212$ : a), b) along the apparent major axis (through two bright knots in the ring); c), d) along the major axis of the central component. Solid line in a) and c) represent the distributions in the $B$ passband, dotted lines in $V$, and dashed ones in the $R$. Solid lines in $\mathbf{b}$ ) and $\mathbf{d}$ ) show the distribution of the $B-V$ color, dashed in the $V-R$ color.

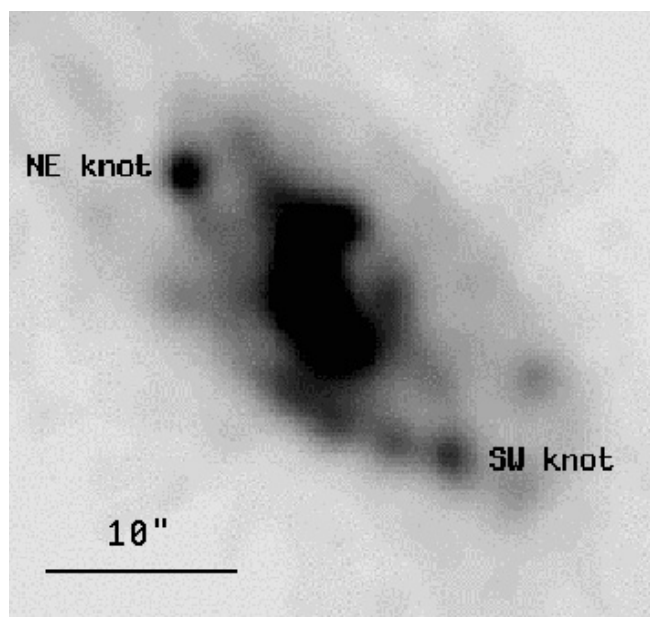

Fig. 4. $B$-band image of A $0017+2212$ after Lucy-Richardson restoration (ten iterations). North is at the top, east to the left.

optical colors of the ring are close to that for whole galaxy: $(B-V)_{0}=+0.48,(V-R)_{0}=+0.38$. In general, the NE arc of the ring is redder $\left((B-V)_{0} \approx+0.55\right)$ than the SW part $\left((B-V)_{0} \approx+0.4\right)$.

Several bright knots are evident in the ring (star clusters, giant HII regions?). The two brightest knots (with $B \approx 21.5-22$ through $2^{\prime \prime}$ circle aperture) are situated near the edges of the ring (Figs. 1 and 4). Optical colors of two brightest knots are somewhat bluer in comparison with nearby parts of the ring: $(B-V)_{0} \approx+0.5$ for the NE knot, and $(B-V)_{0} \approx+0.3$ for the SW (Fig. 4).

\subsubsection{Companion}

The SW companion is faint (total apparent magnitude $B_{\mathrm{T}}=$ $20.0 \pm 0.1$ ) and almost edge-on galaxy (Figs. 1, 3 and 5).

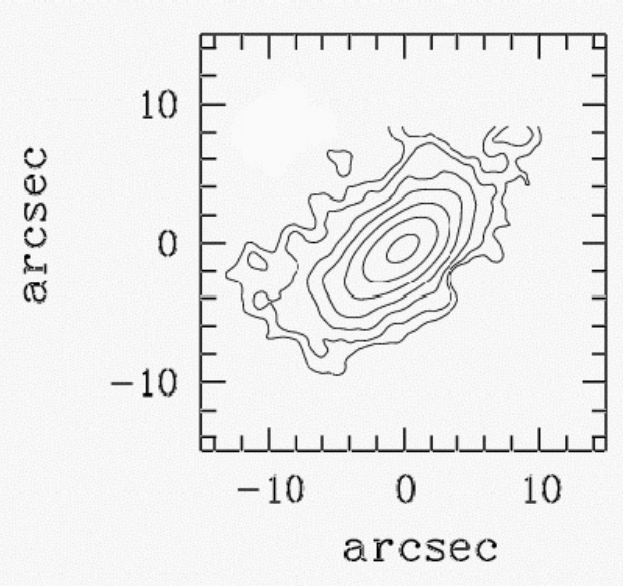

Fig. 5. Isophotal contour image of Anon J001950.0+222814 in the $V$ filter. The fainest contour is $\sim 26.5 \mathrm{mag} \operatorname{arcsec}^{-2}$, isophotes step -0.75 . North is at the top, east to the left.

The galaxy coordinates are $\alpha=00^{\mathrm{h}} 19^{\mathrm{m}} 50.0^{\mathrm{s}}, \delta=+22^{\circ} 28^{\prime} 14^{\prime \prime}$ (J2000) within 1" of error. Therefore, we have denoted this object Anon J001950.0+222814. Integrated optical colors of the galaxy $-(B-V)_{0}=+0.63 \pm 0.10,(V-R)_{0}=+0.48 \pm 0.05-$ are typical for an Sab/Sb spiral (Buta et al. 1994; Buta \& Williams 1995).

The radial surface brightness distribution of Anon J001950.0+ 222814 can be approximated by an exponential law with $\mu_{R, 0}(0)=21.1\left(\mu_{B, 0}(0)=22.1\right)$ and $h=22^{\prime \prime} 25 \pm 0$.' 5 . The radial structure of the disk is strongly asymmetric $-h=2$ '. 75 for the SE part of the disk, $h=1 . .75$ for the NW side.

From the analysis of the vertical structure of the galaxy, we found evidence of a flaring stellar disk. A minor axis photometric cut in the $R$ passband gives an exponential scaleheight value of $h_{z}=0{ }^{\prime} 9$. Cuts at distances $r=2$. .'2-2'.9 from the nucleus give larger values: $h_{z}=1$ '. 1 . The observed $h / h_{z}$ ratio is rather small: 2-2.5. PSF correction increases this ratio to 3-4. Therefore, the stellar disk of Anon J001950.0+222814 is unusually thick in comparison with typical $\mathrm{Sab} / \mathrm{Sb}$ spiral galaxies (e.g. de Grijs 1998; Schwarzkopf \& Dettmar 2000). Such thick stellar disks are typical of interacting galaxies (Reshetnikov \& Combes 1997; Schwarzkopf \& Dettmar 2000).

The southeast part of Anon J001950.0+222814 shows a faint tidal extention pointing to A 0017+2212 (Fig. 5).

\subsubsection{General results on $A 0017+2212$}

In general photometric characteristics (surface brightness distribution, integrated colors (Table 2), color gradients) A 0017+2212 looks similar to late-type spiral galaxies. The main peculiarity of the galaxy is a narrow ring-like structure surrounding central part of the galaxy. This ring is inclined some $30^{\circ}$ with respect to the inner region of the galaxy (Figs. 1, 2 and 4). The galaxy is embedded in a faint and knotty outer envelope. The major axis of the envelope coincides with the major axis of the ring. 
Table 2. General characteristics of PRGs.

\begin{tabular}{|c|c|c|c|c|}
\hline Parameter & $\mathrm{A} 0017+2212$ & UGC 1198 & UGC 4385 & Ref. \\
\hline Morphological type & $\mathrm{Sc} / \mathrm{Scd}$ & Sc: & Im: & \\
\hline Heliocentric velocity $\left(\mathrm{km} \mathrm{s}^{-1}\right)$ & & $1172 \pm 8$ & $1969 \pm 10$ & NED \\
\hline Distance $(\mathrm{Mpc})\left(H_{0}=70 \mathrm{~km} \mathrm{~s}^{-1} \mathrm{Mpc}^{-1}\right)$ & & 19.2 & 26.6 & \\
\hline Major axis, $D_{25}\left(\mu_{B}=25\right)$ & $46^{\prime \prime}$ & $54^{\prime \prime}(5.0 \mathrm{kpc})$ & $70^{\prime \prime}:(9.0 \mathrm{kpc})$ & \\
\hline Axial ratio, $b / a\left(\mu_{B}=25\right)$ & 0.52 & 0.79 & 0.9: & \\
\hline \multicolumn{5}{|l|}{$\begin{array}{l}\text { Total apparent magnitudes } \\
\text { and colors: }\end{array}$} \\
\hline$B_{\mathrm{T}}$ & $16.60 \pm 0.10$ & $14.90 \pm 0.10$ & $14.35 \pm 0.10$ & \\
\hline$(B-V)_{\mathrm{T}}$ & $+0.60 \pm 0.03$ & $+0.70 \pm 0.03$ & $+0.46 \pm 0.04$ & \\
\hline$(V-R)_{\mathrm{T}}$ & $+0.43 \pm 0.03$ & $+0.45 \pm 0.03$ & $+0.34 \pm 0.04$ & \\
\hline$(J-H)_{2 \mathrm{MASS}}$ & $+0.64 \pm 0.09$ & & $+0.52 \pm 0.06$ & {$[1]$} \\
\hline$(H-K)_{2 \mathrm{MASS}}$ & $+0.22 \pm 0.13$ & & $+0.20 \pm 0.09$ & [1] \\
\hline Galactic absorption ( $B$-band) & 0.34 & 0.51 & 0.19 & NED \\
\hline Absolute magnitude, $M_{B, 0}$ & & -17.0 & -18.0 & \\
\hline$(B-V)_{0}$ & +0.52 & +0.58 & +0.41 & \\
\hline$(V-R)_{0}$ & +0.38 & +0.37 & +0.32 & \\
\hline \multicolumn{5}{|l|}{ Exponential disk: } \\
\hline$\mu_{R, 0}(0)$ & 19.6 & 19.1 & 19.3: & \\
\hline$h(R)$ & $4 . .0$ & 6."4 (0.59 kpc) & 7'. 3: (0.94 kpc) & \\
\hline \multicolumn{5}{|l|}{ Ring structure: } \\
\hline$(B-V)_{0}$ & +0.48 & & +0.35 & \\
\hline$(V-R)_{0}$ & +0.38 & & +0.25 & \\
\hline Ring-to-galaxy ratio ( $B$-band) & 0.1 : & & $0.1:$ & \\
\hline Far-infrared luminosity $\left(L_{\odot}\right)$ & & $1.4 \times 10^{9}$ & $4.7 \times 10^{8}$ & NED \\
\hline$M(\mathrm{HI})\left(M_{\odot}\right)$ & & $(1.7 \pm 0.9) \times 10^{8}$ & $1.1 \times 10^{9}$ & {$[2]$} \\
\hline$M\left(\mathrm{H}_{2}\right)\left(M_{\odot}\right)$ & & $(0.8 \pm 0.2) \times 10^{8}$ & & {$[3]$} \\
\hline$M(\mathrm{HI}) / L_{B}\left(M_{\odot} / L_{B, \odot}\right)$ & & 0.17 & 0.45 & \\
\hline$W_{20}\left(\mathrm{~km} \mathrm{~s}^{-1}\right)$ & & $156 \pm 13$ & 196 & {$[2]$} \\
\hline
\end{tabular}

[1] - Skrutskie et al. (1997), [2] - van Driel et al. (2000), [3] - Galletta et al. (1997).

The relatively faint companion (Anon J001950.0+222814) lies $45^{\prime \prime} \mathrm{SW}$ of A 0017+2212. Important features of the companion are:

- asymmetric radial distribution of the surface brightness;

- thick stellar disk;

- tidal tail pointing to A $0017+2212$;

- the companion is located approximately along the major axis of the ring and of the envelope of A $0017+2212$.

All the above features suggest an ongoing interaction of the companion with the main galaxy. One can speculate that we observe the very rare and brief stage of inclined ring formation due to tidal stripping of outer parts of the donor galaxy (Anon J001950.0+222814) (Reshetnikov \& Sotnikova 1997; Bournaud \& Combes 2003). Therefore, A 0017+2212 can be an example of a strongly inclined ring around a spiral host.
Other examples of such objects are NGC 660 (van Driel et al. 1995) and ESO 235-G58 (Iodice 2001).

In an alternative scenario, the observed morphology of A $0017+2212$ is the result of a high-velocity collision between the companion galaxy (Anon J001950.0+222814) and a larger disk system. Therefore, A 0017+2212 can be a "Cartwheeltype" collisional ring galaxy (e.g. Appleton \& Struck-Marcell 1996). It is also possible that A $0017+2212$ is a peculiar ringed spiral with a bar (see examples in Buta \& Combes 1996). In this case the inner structure elongated at $\mathrm{PA} \approx 0^{\circ}$ can be considered as a bar.

Unfortunately, A $0017+2212$ has no published redshift and we cannot describe the galaxy in absolute units. For a crude scaling, we will assume that exponential scalelength of A $0017+2212$ is close to the scale of NGC 660 - the galaxy with inclined ring $\left(h=1.4 \mathrm{kpc}\right.$ for $H_{0}=70 \mathrm{~km} \mathrm{~s}^{-1} \mathrm{Mpc}^{-1}$ 


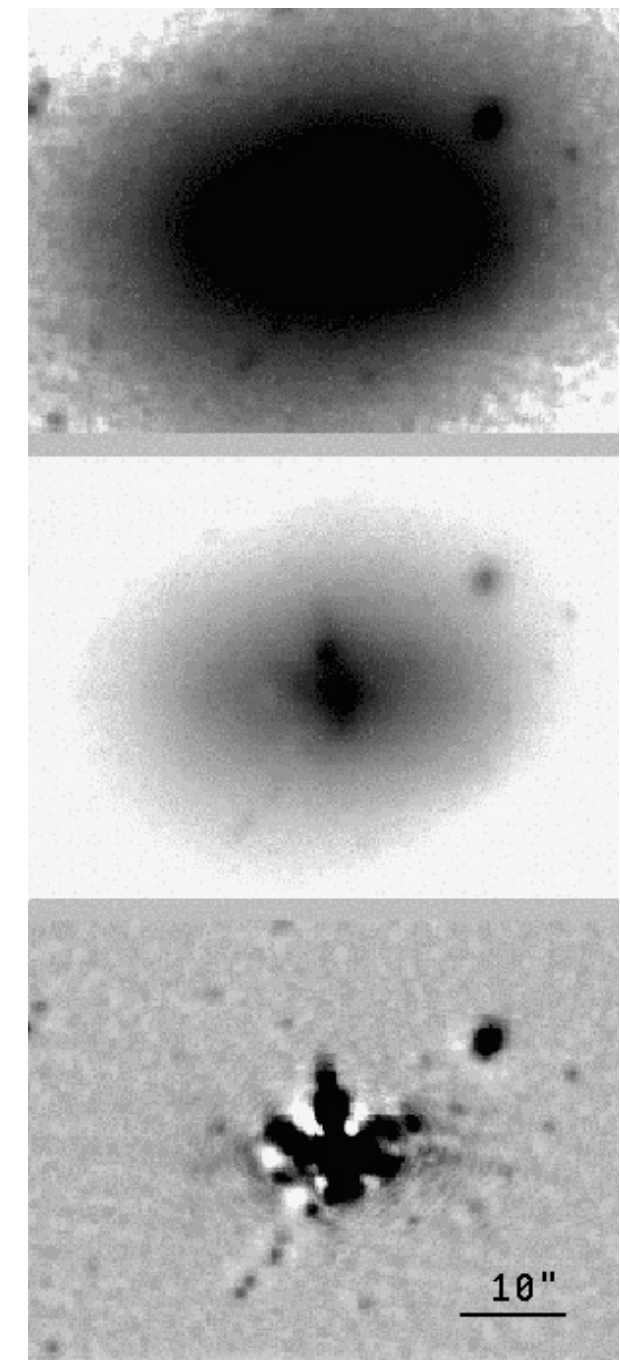

Fig. 6. $R$-band images of UGC 1198 . The first two images are the same frame displayed at different contrasts. Bottom frame is the residual image of the subtraction of the 4". $3 \times 4$ ". 3 median-filtered frame from the original $R$ image. Each image size is $57^{\prime \prime} \times 42^{\prime \prime}$. North is at the top, east to the left.

according to van Driel. 1995). This leads to an absolute magnitude $M_{B, 0}=-18.6$ (close to that for NGC 660) and the ring radius of about $5 \mathrm{kpc}$. The projected distance to the companion thus $21 \mathrm{kpc}$.

Table 2 summarizes the main characteristics of A 0017+2212 and two other PRGs, both found in this work and collected from the literature.

\subsection{UGC 1198}

\subsubsection{Photometric structure}

UGC 1198 demonstrates almost elliptical outer isophotes (Figs. 6 and 7). A faint $\left(B_{\mathrm{T}}=22.6\right)$ elliptical object (background galaxy?) is located $17^{\prime \prime} \mathrm{NW}$ of the galaxy center. UGC 1198 shows disk-like enhancement along the minor axis and two linear structures elongated approximately along the main body of the galaxy (Fig. 6). A faint ring-like feature is seen to the east of the center. The isophotal map (Fig. 7) shows

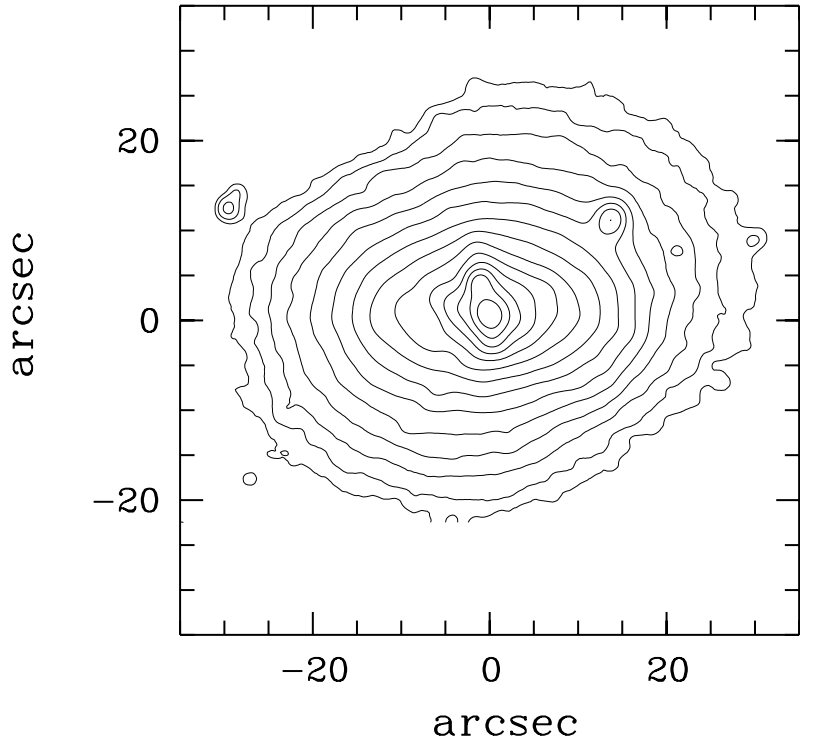

Fig. 7. Isophotal contour image of UGC 1198 in the $V$ passband. The faintest contour is $25.0 \mathrm{mag} \operatorname{arcsec}^{-2}$, isophotes step -0.44 . North is at the top, east to the left.
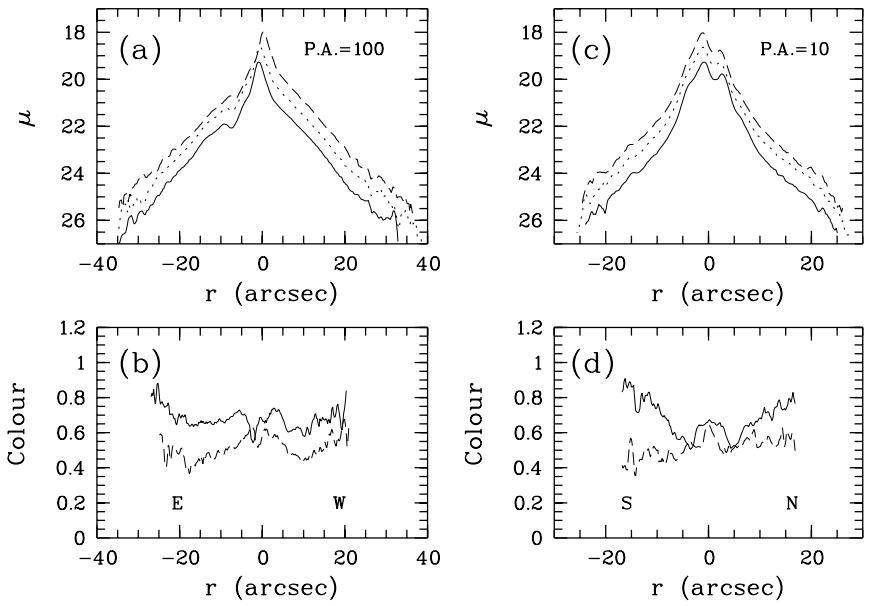

Fig. 8. Photometric profiles for UGC 1198: a), b) along the apparent major axis; c), d) along the major axis of inner elongated component. Solid line in a) and c) represent the distributions in the $B$ passband, dotted lines in $V$, and dashed ones in the $R$. Solid lines in b) and d) show the distribution of the $B-V$ color, dashed in the $V-R$ color.

large-scale twisting: the position angle of the outer isophotes $\left(\mathrm{PA} \approx 115^{\circ}\right)$ is shifted by about $22^{\circ}$ relative to those of the central ones $\left(\mathrm{PA} \approx 93^{\circ}\right)$.

Photometric profiles approximately along the major and minor axes are shown in Fig. 8. Out of the central $5^{\prime \prime}-10^{\prime \prime}$, the surface brightness is nearly exponential with a central surface brightness $\mu_{R, 0}(0)=19.1$ and exponential scalelength $h=6$.' 4 . In the $B$ passband $\mu_{B, 0}(0)=19.8$ that is significantly brighter than typical values for spiral disks. Observed color distributions (Figs. 8b,d) show several blue patches crossing the central region of the galaxy. Another interesting feature is the systematical reddening in the $B-V$ color from the center to the outer parts.

Figure 9 gives the two-dimensional distribution of observed color index $B-V$ in the central region of UGC 1198. A blue 


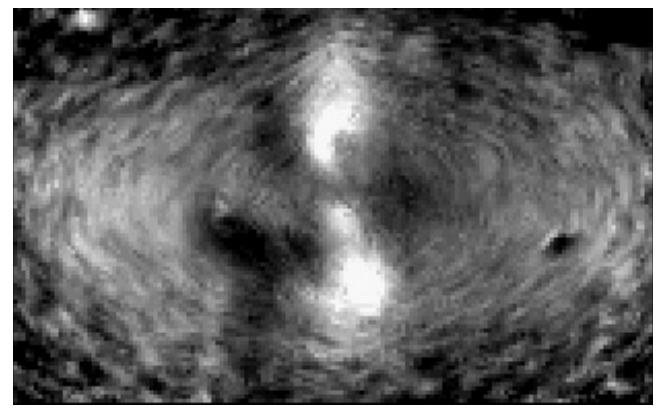

Fig. 9. A gray-scale map of the $B-V$ colour index distribution within central part $\left(31^{\prime \prime} 6 \times 19\right.$ '. 5$)$ of UGC 1198 . The scale is such that dark to light is the sense red to blue. North is at the top and east to the left.

(white in the figure) structure with $(B-V)_{0}=+0.38 \pm 0.05$ is elongated along the minor axis. The observed morphology of this feature suggests that it can be an almost edge-on warped disk or ring. Blue colors give evidence of active star-formation in the disk. The blue structure is crossed by redder patches. Dark regions to the $\mathrm{E}$ and $\mathrm{W}$ of the center in Fig. 9 show $(B-$ $V)_{0} \approx+0.6$. The tangled inner structure of UGC 1198 makes it difficult to discuss it in more detail.

\subsubsection{Fine structures}

In order to emphasize small-scale internal structures of UGC 1198, we present in Fig. 6 (bottom frame) the residual image of the subtraction of a median-filtered image from the original $R$ frame. An inner disk at PA $=10^{\circ}$ is clearly seen. Also, two features elongated at $\mathrm{PA} \approx 70^{\circ}$ and $\approx 110^{\circ}$ intersect the galaxy center.

Some faint point-like sources are seen in the galaxy body (Fig. 6). The brightest sources form a jet-like structure at $\mathrm{PA}=139^{\circ}$. Aperture photometry (diameter of the aperture is 1" 6 ) of 14 clumps gives the following average characteristics: $B_{0}=25.8 \pm 0.4$ or $M_{B, 0}=-5.6,(B-V)_{0}=+0.7 \pm 0.3$, $(V-R)_{0}=+0.7 \pm 0.3$. One can propose that these sources are supergiant stars or compact star clusters.

\subsubsection{Main results on UGC 1198}

This small galaxy demonstrates an exponential surface brightness distribution (Fig. 8) and optical colors typical for a $\mathrm{Sbc/Scd}$ galaxy (Table 2). The detected relative quantities of $\mathrm{HI}$ and $\mathrm{H}_{2}$ (Table 2) also correspond to a late-type spiral galaxy (Young \& Knezek 1989).

The far-infrared luminosity of UGC 1198 estimated from the flux densities at $60 \mu \mathrm{m}$ and $100 \mu \mathrm{m}$ (NED; Lonsdale et al. $1989)$ is $L_{\mathrm{FIR}}=1.4 \times 10^{9} L_{\odot}$. Therefore, the far-infrared to blue luminosity ratio is 2.25 , which is much higher than for isolated spirals (0.4-0.6 - de Jong et al. 1984; Bushouse et al. 1988). Such a high value of the $L_{\mathrm{FIR}} / L_{B}$ ratio is usual for interacting galaxies and for forming PRGs (Bushouse et al. 1988; Reshetnikov \& Combes 1994). The enhanced value of $L_{\mathrm{FIR}} / L_{B}$ gives evidence for active star formation in UGC 1198. The starformation is probably concentrated in a gaseous disk which

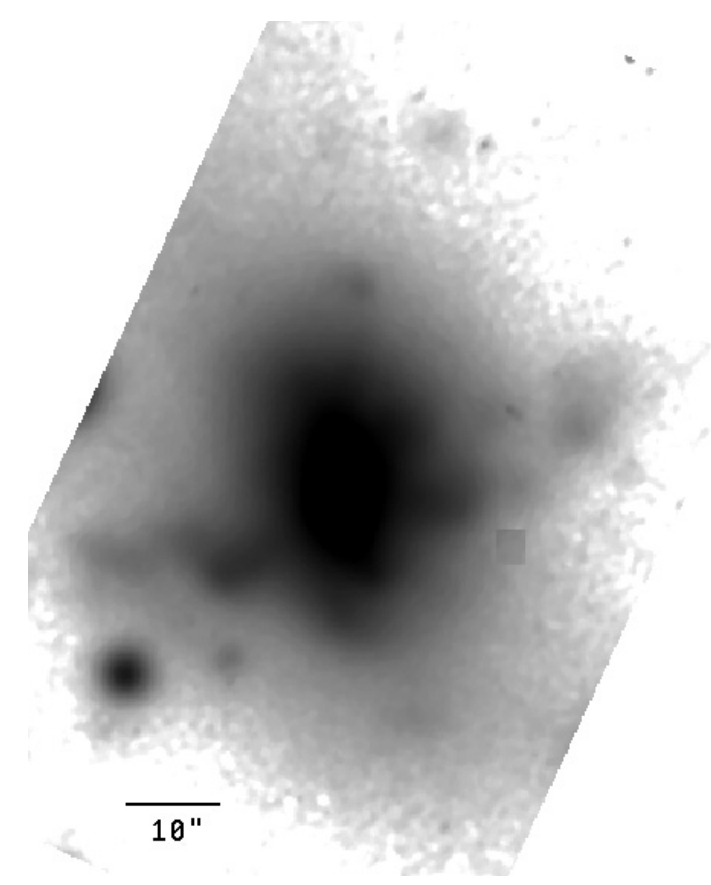

Fig. 10. $B$-band image of UGC 4385 . Image size is $76^{\prime \prime} \times 94^{\prime \prime}$. North is at the top and east to the left.

rotates with $V_{\max } \approx 70 \mathrm{~km} \mathrm{~s}^{-1}$ (Table 2) around the major axis of the galaxy.

The complicated morphology of UGC 1198 suggests that we observe the result of a merger event. One can speculate that UGC 1198 represents an actual collision between a small latetype galaxy and a small early-type galaxy moving on a nearly polar orbit relative to a pre-existing spiral. Tidal debris of the wrecking galaxy has created a red outer envelope and the central part of it is still seen as the object $17^{\prime \prime}$ NW of the UGC 1198 center (Fig. 6).

Final conclusions about the nature of this peculiar galaxy can be obtained from additional high-resolution kinematical observations.

\subsection{UGC 4385}

\subsubsection{General structure}

Optical images of the galaxy (see Figs. 10 and 11) show two distinct morphological components: (1) a central body with a major axis position angle of about $25^{\circ}$, and (2) a polar ring or disk with a mean PA of $\approx 110^{\circ}$. The galaxy is surrounded by a faint envelope whose major axis is aligned with the major axis of the central body. The central body shows clear twisting of the isophotes: from PA close to $0^{\circ}$ near the nucleus to $\mathrm{PA} \approx 25^{\circ}$ at $r \geq 30^{\prime \prime}$ (Fig. 11).

The emission-line rotation curves of UGC 4385 demonstrate that the galaxy's central body rotates around the minor axis while the ring rotates around the major axis (Reshetnikov \& Combes 1994). Therefore, UGC 4385 is one of the few true polar-ring galaxies with a spiral host (the other well-known example is NGC 660 - see van Driel et al. 1995). This conclusion 


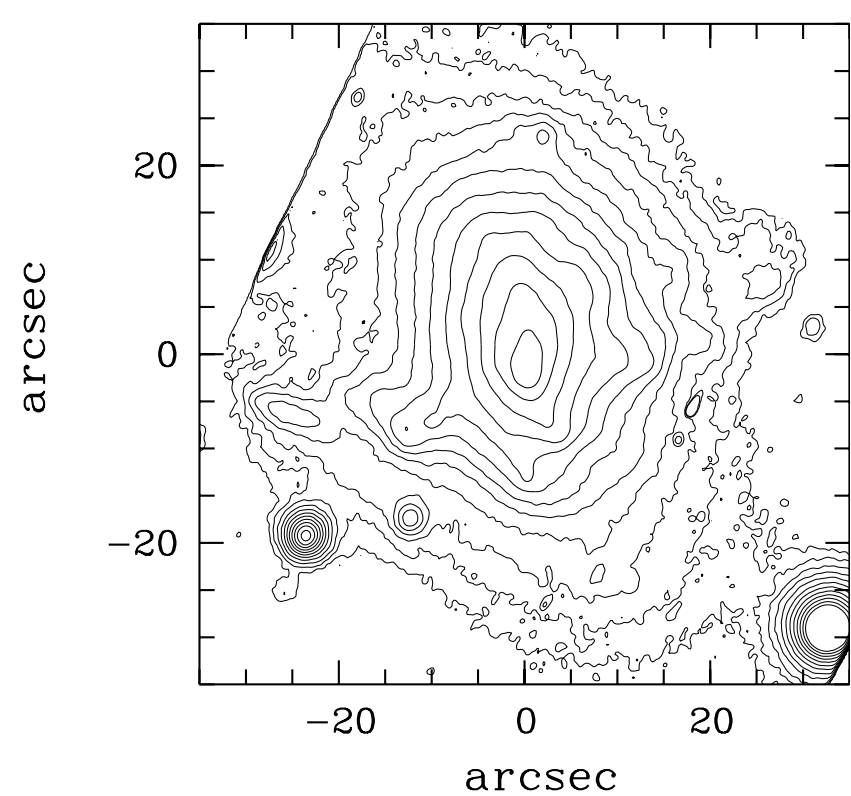

Fig. 11. Isophotal contour image of UGC 4385 in the $V$ passband. The faintest contour is $25.0 \mathrm{mag} \operatorname{arcsec}^{-2}$, isophotes step -0.44 . North is at the top, east to the left.

is based on optical and near-infrared colors, surface brightness distribution and HI content (see discussion below and Table 2).

Figure 12 shows the photometric profiles along the major and minor axes of the galaxy. The central body shows an almost exponential surface brightness distribution along the apparent major axis at $r \leq 20^{\prime \prime}$ (Fig. 12a). At $r \geq 20^{\prime \prime}$ the surface brightness slope becomes less steep, probably due to the outer envelope contribution. The southern part of the major axis profile shows some excess in comparison with the pure exponent (Fig. 12a). The extinction-corrected extrapolated surface brightness of the disk $-\mu_{R, 0}(0)=19.3:\left(\mu_{B, 0}(0)=19.9:\right)-$ is brighter than in typical spiral disks. External parts of the polar ring are clearly seen as a local surface brightness maximum at $r \approx \pm 25^{\prime \prime}$ (Fig. 12c). The major axis profile shows strong color gradient: the central part of the galaxy is rather blue $(B-V \approx+0.35)$, while the outer parts are red $(B-V \approx+0.7)$ (Fig. 12b).

UGC 4385 is a faint source of far-infrared emission (Table 2). The galaxy demonstrates a relatively small $L_{\mathrm{FIR}} / L_{B}$ ratio $(0.32)$ which is typical for unperturbed spirals.

The optical and near-infrared colors of UGC 4385 as far as relative content of $\mathrm{HI}$ (see Table 2) are usual for a late-type disk galaxy.

\subsubsection{Ring}

The ring looks knotty, irregular and slightly inclined to the line of sight. From the apparent axial ratio we esimate the ring's inclination as $80^{\circ}-85^{\circ}$. Also, the ring protrudes to the south (Figs. 10 and 11). The ring is quite extended - its standard isophotal radius $r\left(\mu_{B}=25\right)$ is $30^{\prime \prime}$ or about 4 exponential scalelenghts of the main body.

In Fig. 13 we present the residual image of the galaxy. In the main body we see a strongly warped asymmetric
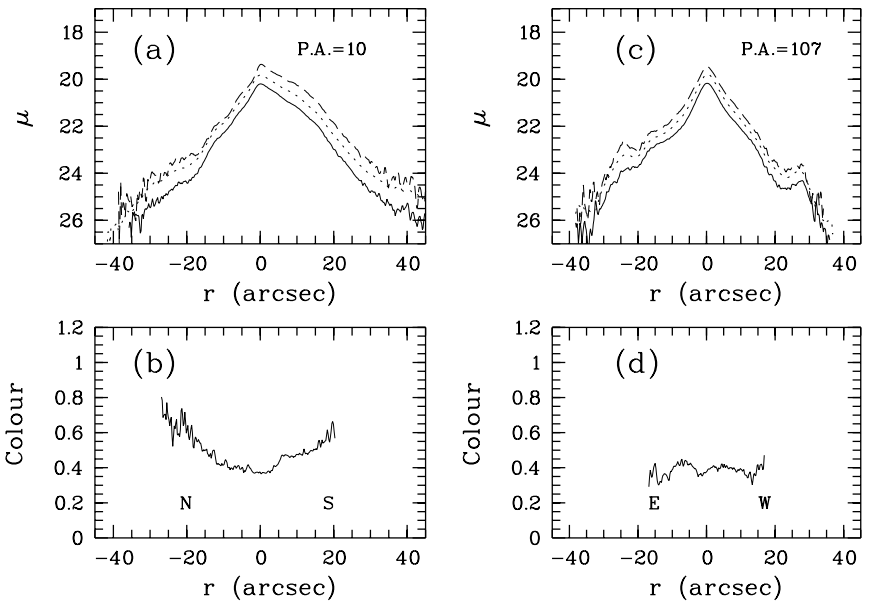

Fig. 12. Photometric profiles for UGC 4385: a), b) along the major axis of central body; c), d) along the major axis of the ring. Solid line in a) and c) represent the distributions in the $B$ passband, dotted lines in $V$, and dashed ones in the $R$. Solid lines in $\mathbf{b}$ ) and d) show the distribution of the $B-V$ color.

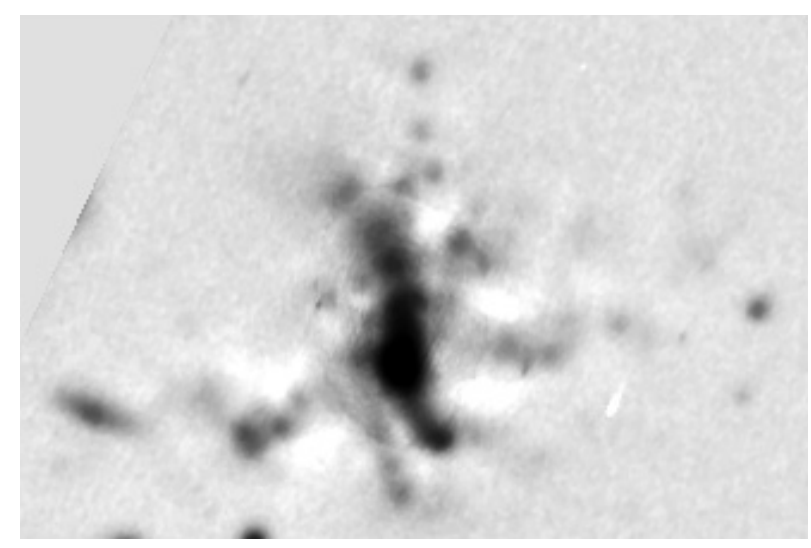

Fig. 13. Residual image of the subtraction of the $8 . .4 \times 88^{\prime \prime} 4$ medianfiltered frame from the original $R$ image. Image size is $69^{\prime \prime} \times 46^{\prime \prime}$. North is at the top, east to the left.

structure with axial ratio $b / a \leq 0.2$ (edge-on disk?). Numerous condensatons are located in the corrugated ring and also are spreaded along the major axis of the central body (Fig. 13). Typical $B$-band apparent magnitudes of the condensations are about $22^{\mathrm{m}} 5-23^{\mathrm{m}}$, which corresponds to an absolute magnitude $M_{B} \approx-9.1--9.6$. Most probably, the condensations represent young star clusters which are very numerous in interacting and merging galaxies (e.g. Schweizer 2002).

Figure 14 shows smoothed emission-line rotation curves of the galaxy with the slit positions along the major axis of the main body and along the polar ring. At $r \geq 14^{\prime \prime}(\geq 1.8 \mathrm{kpc})$ the observed polar and equatorial (along the galaxy's major axis) velocities are almost equal. At the last point where measurements along two ortoghonal directions are available $\left(r=26{ }^{\prime} \cdot 2=3.6 h=3.4 \mathrm{kpc}\right)$, the observed $V_{\mathrm{pol}} / V_{\mathrm{eq}}$ ratio is $1.04 \pm 0.22$. After applying a small and rather uncertain correction for non-edge-on orientation of the central body and the ring, this ratio will stay close to 1 . This gives an argument in favour of an almost spherical dark halo in UGC 4385 but, within quoted errors, even flattened halo is consistent with 


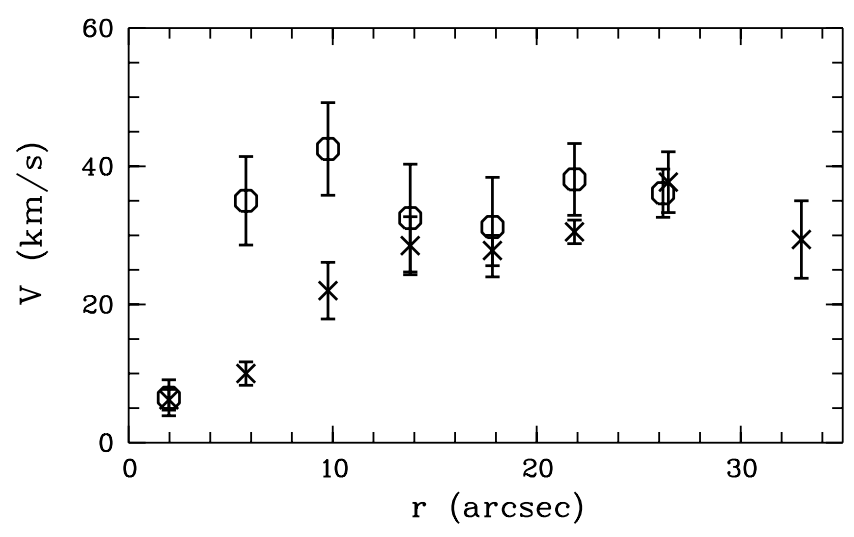

Fig. 14. Averaged $\mathrm{H} \alpha$ rotation curve of the main body of UGC 4385 at $\mathrm{PA}=17^{\circ}$ (open circles) and of the polar ring at $\mathrm{PA}=107^{\circ}$ (crosses). Original observations were presented in Reshetnikov \& Combes (1994).

observations. For instance, assuming a model with a scalefree potential (see Whitmore et al. 1987), from the observed $V_{\text {pol }} / V_{\text {eq }}$ ratio one can obtain that the flattening of equipotentials in the galaxy is $1.05 \pm 0.27$.

Assuming a spherical mass distribution, the total mass to $B$-luminosity ratio within the last point of the rotation curve of the ring $\left(r=33^{\prime \prime}\right)$ is $0.4 \pm 0.15$ (in solar units).

\subsubsection{Main results on UGC 4385}

Kinematical evidence for two luminous nearly orthogonal components and the optical morphology suggest that UGC 4385 is true polar-ring galaxy. The chaotic appearance of the ring, its protuberant shape, global assymetry of the galaxy, strong isophotal twisting all suggest the relative youth of the object. Comparison with results of numerical simulations (e.g. Bekki 1997; Bournaud \& Combes 2003) shows that in the case of UGC 4385 we can observe an early stage of PRG formation due to galaxy merging. Also, there are no comparably sized companions (possible donors for the accretion scenario) at least up to $50 \mathrm{kpc}$ from the galaxy. Most probably, we observe a headon collision between two small galaxies. At least one of the galaxies is a late-type galaxy and we see its perturbed disk as the polar ring. A red stellar halo is a natural consequence of the merging process (Bournaud \& Combes 2003). Numerous candidates for young star clusters (Fig. 13) also support possible ongoing interaction of two pre-existing galaxies.

The maximum rotation velocity of the galaxy derived from the HI width (Table 2) is $\approx 80 \mathrm{~km} \mathrm{~s}^{-1}$ that is in evident contradiction with optical rotational velocity $\left(V_{\max } \approx 40 \mathrm{~km} \mathrm{~s}^{-1}-\right.$ Fig. 14). It means, probably, that the HI disk of the galaxy is more extended than the optical image and that real rotation curve is still rising beyond the optical disk. As in the cases of A 0017+2212 and UGC 1198, new kinematical data and detailed numerical simulations are highly needed for better understanding of this galaxy.

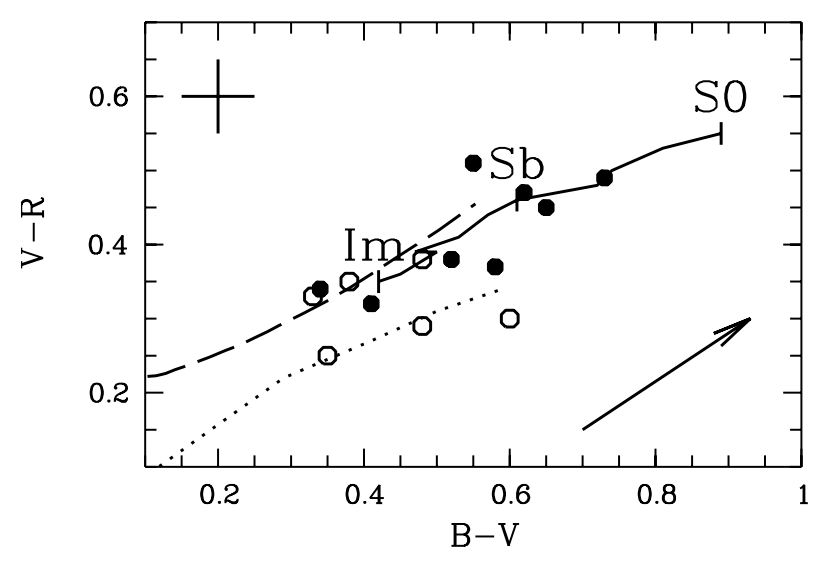

Fig. 15. $B-V$ vs. $V-R$ diagram for the main galaxies (solid circles) and the polar rings (open circles) of PRGs with spiral hosts. Solid line represents observational relationship for normal Im-S0 galaxies according to Buta et al. (1994) and Buta \& Williams (1995). Dotted line - simple stellar populations models with different metallicities at $1 \mathrm{Gyr}$ age (Kurth et al. 1999). Metallicity varies from $Z=0.0001$ at bottom left end of the line to $Z=0.02$ at top right. Dashed line shows evolutionary track for the stellar synthesis model optimized for a latetype galaxy (Fioc \& Rocca-Volmerange 1997). Arrow indicates the value and direction of a reddening for a total $B$-band absorption of $1^{\mathrm{m}}$, cross demonstrates typical error of colors measurements.

\section{Photometric features of PRGs with spiral hosts}

In this section we will discuss available photometric characteristics for the PRGs with spiral central galaxies. As for "classical" PRGs with early-type gas-free hosts, the main observational features were summarized by Reshetnikov \& Sotnikova (1997) and by Iodice et al. (2002a,b).

Among relatively regular PRGs with optical rings we have selected three galaxies with spiral hosts: NGC 660 (van Driel et al. 1995; Benvenuti et al. 1976), ESO 235-G58 (Buta \& Crocker 1993; Iodice 2001), UGC 4385. Several other objects can be classified as forming rings around (or inside?) spiral galaxies due to external accretion or merging: NGC 3808B, NGC 6286 (Reshetnikov et al. 1996), UGC 4261 (Reshetnikov et al. 1998), UGC 5600 (Karataeva et al. 2001), A 0017+2212, UGC 1198 from the present work. We will consider also NGC 2748 - a spiral with a very smooth and faint polar ring (Hagen-Thorn et al. 1996).

\subsection{Central galaxies}

The mean $B$-band absolute magnitude of the sample PRGs is between -19 . 0 and -19 . 5 (without or with internal extinction corrections) with a standard deviation of $1 \mathrm{~m}$. 2 . Therefore, they are sub- $L^{*}$ spirals, on average.

Figure 15 shows the observed integrated colors of the host galaxies in the two-color diagram. It is evident in the figure that the optical colors of the spiral hosts appear very similar to those of normal spiral galaxies. After correction for internal extinction (several sample galaxies are almost edge-on disks) 
according to the RC3 (de Vaucouleurs et al. 1991) prescriptions, we obtain the next average color indices:

$\langle B-V\rangle=+0.53 \pm 0.11(10$ objects $)$,

$\langle V-R\rangle=+0.38 \pm 0.05$ ( 8 objects $).$

Such colors are usual for normal Sbc-Sc spiral galaxies (Buta et al. 1994; Buta \& Williams 1995).

In the same figure we present evolutionary track for a model spiral galaxy computed with the PEGASE. 2 code (Fioc \& Rocca-Volmerange 1997). The model includes the following main ingredients: extended infall of a zero-metallicity gas (infall timescale is $5 \mathrm{Gyr}$ ); a gas-dependent star formation rate; inclination-averaged extinction for the disk geometry. The track (dashed line) starts from 1 Gyr age at the left side and finishes at 14 Gyr. This model satisfactorily reproduces the photometric properties of late-type spirals in the local universe as far as spiral hosts of PRGs. The implied model age of the main galaxies is $\geq 7 \mathrm{Gyr}$. The average extinction-corrected colors corresponds to $12 \pm 2$ Gyr age.

The mean extrapolated central surface brightness of an exponential disk in the $B$ passband (in this filter the data is available or can be estimated from published results for all 10 galaxies) is $\mu_{0}(B)=20.2 \pm 0.9$. This is the mean observational value, not corrected for inclination effects. Adopting the extreme correction for a fully transparent galaxy (evidently, this is not correct for the $B$ filter), we obtain the corrected value of $\mu_{0}^{\mathrm{c}}(B)=21.0 \pm 1.2$. Therefore, stellar disks of spiral PRGs are $0 \mathrm{~m} .7-1 \mathrm{~m} .5$ brighter in comparison with normal non-dwarf galaxies (Freeman 1970). The same result was found earlier for the disks of early-type PRGs (Reshetnikov et al. 1994; Iodice et al. 2002b). Comparing the PRGs characteristics with surveys of local spirals (e.g. O'Neil et al. 2003), we have found that such bright disks are about 10 times less frequent than "Freeman" disks with $\mu_{0}(B)=21.7$.

The probable reason for bright disks in the PRGs is active star formation triggered by past or current external accretion. Other manifestation of recent interaction in spiral PRGs is the unusual vertical structure of their disks. For instance, two spiral PRGs with almost edge-on main galaxies (NGC 3808B and NGC 6286) show flaring and very thick (with $h / z_{0}$ ratio about 2-3) stellar disks (Reshetnikov et al. 1996).

The average value of the exponential scalelength in the $B$ filter is $\langle h\rangle=1.9 \pm 1.2 \mathrm{kpc}$. Spiral galaxies with such disks are among the most frequent in the local universe (de Jong 1996).

\subsection{Polar structures}

Typical luminosity of off-plane optical structures is about $0.1-0.2$ of the luminosity of spiral hosts. For NGC 660 and ESO 235-G58 the ring-to-host galaxy ratio rises to $\sim 1$ in the $B$-passband.

The average observed colors of polar structures are

$\langle B-V\rangle=+0.44 \pm 0.10$ (6 objects),

$\langle V-R\rangle=+0.33 \pm 0.05$ (7 objects $).$
Therefore, the polar structures of spiral PRGs, on average, have bluer colors than the host galaxies but the difference is not very large (Sect. 4.1). As one can see in Fig. 15, several polar structures demonstrate optical colors usual for late spirals and for spiral arms of galaxies. In other cases (NGC 2748, NGC 6286, UGC 4385) the colors are shifted down from the normal color dependence. The interpretation of the two-color diagram depends on the adopted metallicity and star formation history in the polar structures. For illustrative purposes we present in Fig. 15 the colors of a simple stellar population model with the age of 1 Gyr (Kurth et al. 1999). The colors of off-plane structures in NGC 2748, NGC 6286, UGC 4385 are consistent with this model in the range of metallicities from $Z=0.0004$ to $Z=0.02$.

\section{Tully-Fisher relation for PRGs}

The Tully-Fisher (TF) relation is one of the most important scaling relations for spiral galaxies and it also provides constraints on galaxy formation. In a recent work, Iodice et al. (2003) have concluded that the PRG distribution in the TF diagram does not coincide with the loci occupied by normal disk galaxies. (For previous attempts to consider the TF relation for PRGs see Knapp et al. 1985; Reshetnikov et al. 2001.) Iodice et al. have concluded that this observational evidence can be explained by a flattened dark halo, aligned with the polar ring. In this secton, we will consider the TF relation using a large sample of PRGs (including the new objects presented in this work) and considering different groups of galaxies separately.

\subsection{Comparison of the $\mathrm{HI}$ widths and velocities}

In order to enlarge the available kinematical data we will combine the HI profile widths with rotational velocities measured from optical emission lines. As a first step, we decided to compare the observed maximum rotation velocities obtained from emission-line rotation curves $\left(V_{\max }\right)$ with the HI global profile widths ( $W_{20}-$ measured HI width at $20 \%$ of peak intensity). To make a fair comparison, we have corrected original $W_{20}$ values for the instrumental broadening (following Bottinelli et al. 1990) and for random motions (according to the prescription of Tully \& Fouque 1985). For the last correction we have adopted characteristic parameter values $W_{\mathrm{c}}=120 \mathrm{~km} \mathrm{~s}^{-1}$ and $W_{\mathrm{t}}=22 \mathrm{~km} \mathrm{~s}^{-1}$ (Rhee 1996; Verheijen 1997).

Figure 16 shows the comparison of the corrected $W_{20}$ values (denoted as $W_{R}$ ) with observed maximum rotational velocities from optical rotation curves. Our sample of PRGs includes 21 galaxies from the PRC with available HI and optical kinematical data. The HI data were taken mainly from van Driel et al. $(2000,2002)$. In the case of multiple measurements we preferred the Nacay observations. Optical maximum rotation velocities were taken mainly from the data published in Reshetnikov \& Combes (1994). For several additional objects the data are from the PRC (A-3, B-19, B-21), Whitmore et al. (1987) (A-1, A-2, A-5), van Driel et al. (1995) (C-13), Hagen-Thorn \& Reshetnikov (1997) (B-3), Reshetnikov et al. (2001) (B-18). 


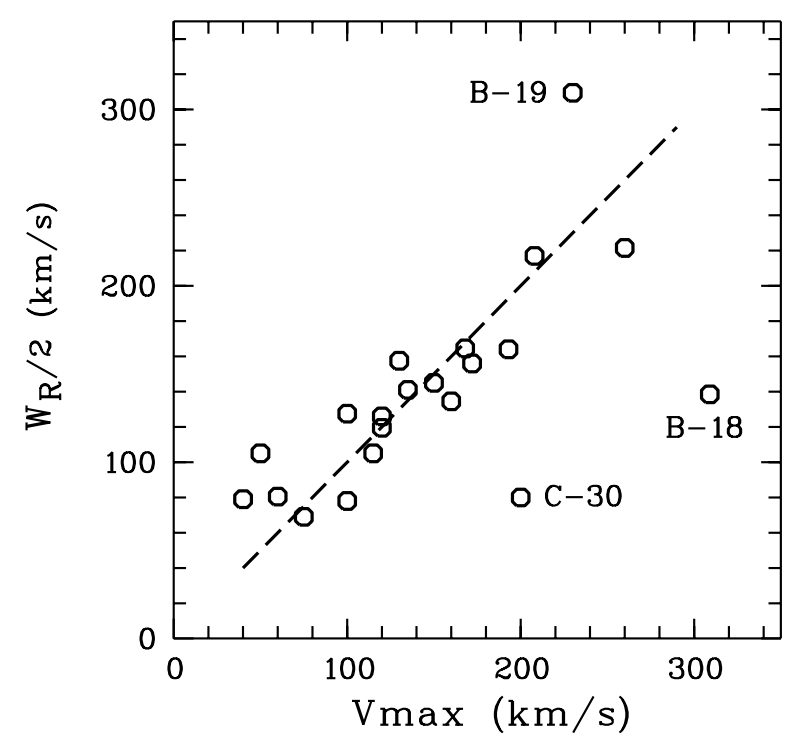

Fig. 16. Comparison of the corrected global HI profile widths with $V_{\max }$ values. Dashed line shows $W_{R} / 2=V_{\max }$ relation.

After rejecting the three extreme outliers (see Fig. 16), the mean difference between $W_{R} / 2$ and $V_{\max }$ is $+2 \pm 25 \mathrm{~km} \mathrm{~s}^{-1}$ (18 galaxies). Excluding dwarf objects with $V_{\max }<60 \mathrm{~km} \mathrm{~s}^{-1}$, we obtain $\left\langle\frac{W_{R}}{2}-V_{\max }\right\rangle=-4 \pm 20 \mathrm{~km} \mathrm{~s}^{-1}$ (16 galaxies). These results show that optical and HI rotation velocities are wellcorrelated and therefore internal kinematics measured from optical emission lines and from HI data should follow the same TF relation.

As for the outliers, in all three cases the HI data are uncertain. For instance, AM 1934-563 (B-18) is a member of a triple system and its HI profile may be confused with another member of the group (van Driel et al. 2002). The $W_{20}$ value for AM 2020-504 (B-19) is marked by van Driel et al. (2002) as uncertain. The UGC 5101 (C-30) HI profile (it is in absorption) could be an off-band detection (van Driel et al. 2000).

\subsection{TF relation}

\subsubsection{PRGs}

Figure 17a shows the Tully-Fisher relation for true polar-ring galaxies (see description of Category A objects in the PRC). We have considered two groups of objects. The first group consists of galaxies with extended polar rings. This group includes A 0136-0801, UGC 7576, NGC 4650A, UGC 9796, NGC 660, NGC 5122, ESO 503-G17, and ESO 235-G58. The first six galaxies are well-known PRGs. ESO 503-G17 (B-12) is an excellent candidate PRG whose optical morphology closely resembles typical PRGs (see PRC). ESO 235-G58 is also an excellent candidate described in detail by Buta \& Crocker (1993) and by Iodice (2001). The second group consists of objects with relatively short rings: ESO 415-G26, NGC 2685, IC 1689 , AM 2020-504, ESO 603-G21, and UGC 4385.

The $B$-band magnitudes of the galaxies were taken mainly from original works with results of detailed surface photometry (see references in van Driel et al. 2000 and in the present work). For A 0136-0801, ESO 503-G17, ESO 415-G26 and
AM 2020-504 we used the PRC data, and for NGC 5122 the magnitude from the NED. The maximum rotation velocities were estimated from published $W_{20}$ values (see previous section). For AM 2020-504 with uncertain HI data (van Driel et al. 2002) we used the $V_{\max }$ value from the optical rotation curve of the ring component presented in the PRC. Note that the $\mathrm{HI}$ in classic PRGs associates with the polar ring and, therefore, the observed HI widths trace the dynamics of the polar structure.

In agreement with Iodice et al. (2003), the PRG characteristics show a significant shift from the TF relation for normal spirals (Fig. 17a). This effect is cleary seen for PRGs with extended and short rings. Only one galaxy - UGC 4385 - lies on the TF relation. How can possible internal absorption in the main galaxies (for spiral hosts of NGC 660 and ESO 235-G58) and in the rings influence the TF relation for PRGs? We have corrected the total magnitudes for the absorption following Tully et al. (1998). As a result, two other galaxies NGC 4650A and ESO 235-G58 - move exactly to the TF. The rest of PRGs (11 objects) lie at lower velocities (for a given luminosity) than those predicted by the TF relation.

Comparing the PRG location with the relations for normal spirals from Tully et al. (1998) and Kannappan et al. (2002), we have found that true PRGs demonstrate 35\% $\pm 10 \%$ larger rotation velocities than disk galaxies. Excluding UGC 4385, NGC 4650A, and ESO 235-G58, we obtain the mean difference of $45 \% \pm 10 \%$. According to Iodice et al. (2003), these larger velocities can be reproduced in 2D and 3D $N$-body models when the dark halo is flattened towards the polar plane. This result is of primary importance since, if confirmed, it would imply that some of the dark matter is dissipative, and set strong constrains on dark matter candidates.

For galaxies on the TF relation (UGC 4385, NGC 4650A, ESO 235-G58), it is interesting to note that the rings in all three galaxies are bluer, on average, in comparison with the ring components in the rest of the objects $(B-V \approx+0.3$ vs. $B-V \approx+0.5$ ). One can suppose that these rings are still in the formation process (see, for instance, Sect. 3.3.3).

\subsubsection{Forming PRGs}

Figure $17 \mathrm{~b}$ presents the data for 20 objects from the PRC with available kinematic and photometric data. The objects are: PRC B-1, 2, 6, 8, 9, 11, 17, 18; C-7, 8, 12, 24, 25, 28, 30, 46, 51; D-12, 18, 19. Photometric measurements were taken from original works and from the NED. For most of the objects we used the HI measurements for the rotation velocities estimations (Sect. 5.1), while for AM 1934-563, UGC 5101, UGC 4323, and ESO 576-G69 we extracted $V_{\max }$ values from optical rotation curves. For evident spiral galaxies with non-face-on orientation (e.g. NGC 3808B, NGC 6286, AM 1934-563, NGC 2748) we have corrected the apparent magnitudes for internal absorption (Tully et al. 1998). From the morphological point of view, most of the objects are interacting galaxies or mergers and so we have called the sample forming PRGs.

As it is evident in the figure, forming PRGs follow the standard TF relation but with rather large scatter. The large scatter 

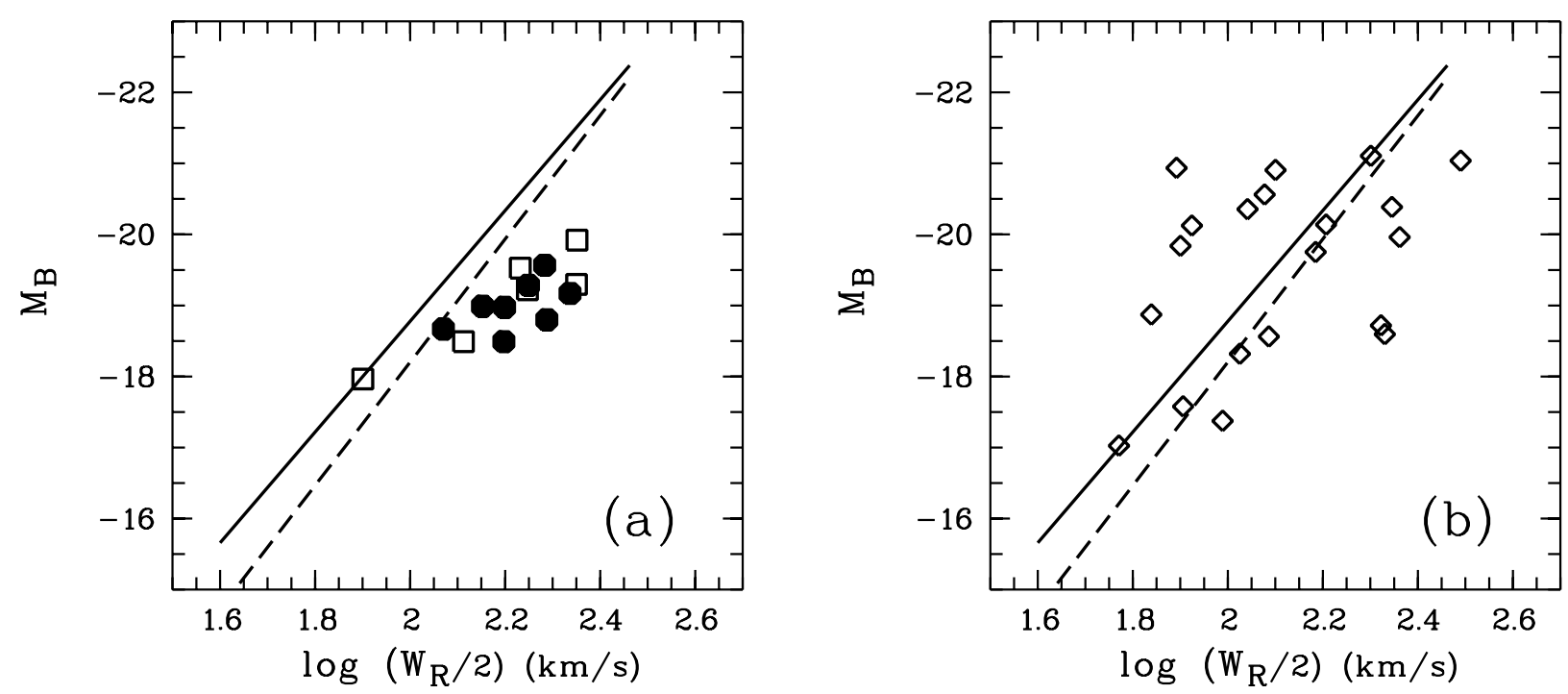

Fig. 17. Absolute $B$-band magnitude vs. the maximum rotation velocity for PRGs: a) for true kinematically-confirmed PRGs (solid circles galaxies with extended rings, squares - with short rings); b) for forming PRGs (rhombs). Solid and dashed lines show the TF relation for spiral galaxies according to Tully et al. (1998) and Kannappan et al. (2002) correspondingly $\left(H_{0}=70 \mathrm{~km} \mathrm{~s}^{-1} \mathrm{Mpc}^{-1}\right)$.

can be explained in part by the very uncertain geometry of the objects. This leads to very uncertain kinematic and photometric inclination corrections. Also, in many objects the $\mathrm{HI}$ is in the host galaxies and in possible rings. So we cannot expect that HI widths trace the dynamics of the polar structure only.

\section{Conclusions}

In this work we present new $B, V, R$ photometric data for three objects from the PRC (A 0017+2212, UGC 1198, UGC 4385). It was shown that all three galaxies are late-type spiral or irregular galaxies. Main observational peculiarities of the objects can be probably explained by current or recent external accretion or gravitational interaction with a close companion.

Comparison with other PRGs with spiral central galaxies yields that the host galaxies are, on average, normal sub- $L^{*}$ Sbc-Sc spirals with bright exponential disks. The offplane optical structures have somewhat bluer colors than the host galaxies.

We have confirmed recent result by Iodice et al. (2003) that true evolved polar-ring galaxies with short or extended rings show larger maximum rotation velocities than expected for the observed optical luminosity.

Acknowledgements. I would like to thank the anonymous referee for useful comments. I acknowledge support from the Russian Foundation for Basic Research (03-02-17152) and from the Russian Federal Program "Astronomy" (40.022.1.1.1101).

\section{References}

Appleton, P. N., \& Struck-Marcell, C. 1996, Fund. Cosm. Phys., 16, 111

Bekki, K. 1997, ApJ, 490, L37

Bekki, K. 1998, ApJ, 499, 635

Benvenuti, P., Capaccioli, M., \& D’Odorico, S. 1976, A\&A, 53, 141
Borisenko, A. N., Markelov, S. V., \& Ryadchenko, V. P. 1991, Prepr. SAO $N 76$

Bottinelli, L., Gouguenheim, L., Fouque, P., \& Paturel, G. 1990, A\&AS, 82, 391

Bournaud, F., \& Combes, F. 2003, A\&A, 401, 817

Bushouse, H. A., Lamb, S. A., \& Werner, M. W. 1988, ApJ, 335, 74

Buta, R., \& Crocker, D. A. 1993, AJ, 106, 939

Buta, R., Mitra, S., de Vaucouleurs, G., \& Corwin, H. G. 1994, AJ, 107,118

Buta, R., \& Williams, K. L. 1995, AJ, 109, 543

Buta, R., \& Combes, F. 1996, Fund. Cosm. Phys., 17, 95

de Jong, T., Clegg, P. E., Soifer, B. T., et al. 1984, ApJ, 278, L67

de Jong, R. S. 1996, A\&A, 313, 45

de Grijs, R. 1998, MNRAS, 299, 595

de Vaucouleurs, G., de Vaucouleurs, A., Corwin, H. G., et al. 1991, Third Reference Catalogue of Bright Galaxies (Springer-Verlag)

Fioc, M., \& Rocca-Volmerange, B. 1997, A\&A, 326, 950

Freeman, K. C. 1970, ApJ, 160, 811

Galletta, G., Sage, L. J., \& Sparke, L. S. 1997, MNRAS, 284, 773

Hagen-Thorn, V. A., Reshetnikov, V. P., \& Yakovleva, V. A. 1996, Astron. Zh., 73, 36 (Engl. transl. in Astron. Rep.)

Hagen-Thorn, V. A., \& Reshetnikov, V. P. 1997, A\&A, 319, 430

Iodice, E. 2001, Ph.D. Thesis, SISSA, Trieste

Iodice, E., Arnaboldi, M., Sparke, L. S., et al. 2002a, A\&A, 391, 103 Iodice, E., Arnaboldi, M., Sparke, L. S., \& Freeman, K. C. 2002b, A\&A, 391, 117

Iodice, E., Arnaboldi, M., Bournaud, F., et al. 2003, ApJ, 585, 730

Kannappan, Sh. J., Fabricant, D. G., \& Franx, M. 2002, AJ, 123, 2358

Karataeva, G. M., Yakovleva, V. A., \& Hagen-Thorn, V. A. 2001, Pis'ma v Astron. Zh., 27, 94 (Engl. transl. in Astron. Let.)

Kinney, A. L., Gallagher, J., Matthews, L., et al. 1999, AAS, 194.0601

Knapp, G. R., van Driel W., \& van Woerden, H. 1985, A\&A, 142, 1

Kurth, O. M., Fritze-v.Alvensleben, U., \& Fricke, K. J. 1999, A\&AS, 138,19

Landolt, A. U. 1983, AJ, 88, 439

Lonsdale, C. J., Helou, G., Good, J. C., et al. 1989, Catalogued Galaxies and Quasars in the IRAS Survey, 2nd version, (Pasadena: JPL)

O'Neil, K., Andreon, S., \& Cuillandre, J.-C. 2003, A\&A, 399, L35 
Reshetnikov, V. P., Hagen-Thorn, V. A., \& Yakovleva, V. A. 1993, Skrutskie, M. F., Schneider, S. E., Stiening, R., et al. 1997, in The A\&A, 278, 351

Reshetnikov, V. P., \& Combes, F. 1994, A\&A, 291, 57

Reshetnikov, V. P., Hagen-Thorn, V. A., \& Yakovleva, V. A. 1994, A\&A, 290, 693

Impact of Large Scale Near-IR Sky Surveys, ed. F. Garzon, et al. (Dordrecht, Kluwer), 25

Smith, P. S., Januzzi, B. T., \& Elston, R. 1991, ApJS, 77, 67

Tully, R. B., \& Fouque, P. 1985, ApJS, 58, 67

Reshetnikov, V. P., Hagen-Thorn, V. A., \& Yakovleva, V. A. 1996, A\&A, 314, 729

Reshetnikov, V., \& Combes, F. 1997, A\&A, 324, 80

Reshetnikov, V., \& Sotnikova, N. 1997, A\&A, 325, 933

Reshetnikov, V. P., Hagen-Thorn, V. A., \& Yakovleva, V. A. 1998, Astron. Zh., 75, 498 (Engl. transl. in Astron. Rep.)

Tully, R. B., Pierce, M. J., Huang, J.-Sh., et al. 1998, AJ, 115, 2264

van Driel, W., Combes, F., Casoli, F., et al. 1995, AJ, 109, 942

van Driel, W., Arnaboldi, M., Combes, F., \& Sparke, L. S. 2000, A\&AS, 141, 385

van Driel, W., Combes, F., Arnaboldi, M., \& Sparke, L. S. 2002, A\&A, 140, 2002

Reshetnikov, V. P., Faundez-Abans, M., \& de Oliveira-Abans, M. 2001, MNRAS, 322, 689

Rhee, M.-H. 1996, Ph.D. Thesis, Univ. Groningen

Schlegel, D. J., Finkbeiner, D. P., \& Davis, M. 1998, ApJ, 500, 525

Schwarzkopf, U., \& Dettmar, R.-J. 2000, A\&A, 361, 451

Verheijen, M. A. W. 1997, Ph.D. Thesis, Univ. Gronongen

Whitmore, B. C., McElroy, D. B., \& Schweizer, F. 1987, ApJ, 314, 439

Whitmore, B. C., Lucas, R. A., McElroy, D. B., et al. 1990, AJ, 100, 1489 (PRC)

Schweizer, F. 2002, in New Horizonts in Globular Cluster Astronomy, Young, J. S., \& Knezek, P. M. 1989, ApJ, 347, L55 ed. G. Piotto et al., in press [astro-ph/0212243] 